\title{
On Discovery of Extremely Low-Dimensional Clusters using Semi-Supervised Projected Clustering
}

\author{
Kevin Y. Yip, David W. Cheung \\ Department of Computer Science \\ University of Hong Kong \\ \{ylyip,dcheung\}@cs.hku.hk
}

\author{
Michael K. Ng \\ Department of Mathematics \\ University of Hong Kong \\ mng@maths.hku.hk
}

\begin{abstract}
Recent studies suggest that projected clusters with extremely low dimensionality exist in many real datasets. A number of projected clustering algorithms have been proposed in the past several years, but few can identify clusters with dimensionality lower than $10 \%$ of the total number of dimensions, which are commonly found in some real datasets such as gene expression profiles. In this paper we propose a new algorithm that can accurately identify projected clusters with relevant dimensions as few as 5\% of the total number of dimensions. It makes use of a robust objective function that combines object clustering and dimension selection into a single optimization problem. The algorithm can also utilize domain knowledge in the form of labeled objects and labeled dimensions to improve its clustering accuracy. We believe this is the first semi-supervised projected clustering algorithm. Both theoretical analysis and experimental results show that by using a small amount of input knowledge, possibly covering only a portion of the underlying classes, the new algorithm can be further improved to accurately detect clusters with only $1 \%$ of the dimensions being relevant. The algorithm is also useful in getting a target set of clusters when there are multiple possible groupings of the objects.
\end{abstract}

\section{Introduction}

Recently many studies have suggested the presence of low dimensional clusters in high-dimensional real datasets. For example, in a typical microarray gene expression dataset that contains the expression values of several thousands of genes in different samples, it is common to find that only several tens of genes have expression patterns unique to each cluster of samples [14]. The genes are called the relevant genes, as opposed to the irrelevant genes that do not help much in identifying the cluster members. Due to the large number of genes being irrelevant to each cluster, two samples in the same cluster could have low similarity when measured by a similarity function that considers the expression values of all genes. The clusters are thus undetectable by traditional clustering algorithms. The same kind of low dimensional clusters could also exist in datasets from various domains such as computer vision [15], e-commerce [19], text mining and nutrition value analysis [24].

The projected clustering problem [1] is defined for such a scenario. Each projected cluster is a set of member objects with an associated set of relevant dimensions such that the member objects are similar to each other in the subspace formed by the relevant dimensions, but dissimilar to objects outside the cluster. In this paper we measure object similarity based on Euclidean distance, so a dimension is more relevant to a cluster if the projections of its members on the dimension are closer to each other, but more remote from the projections of other objects. The goal of a projected clustering algorithm is to identify clusters of objects and their relevant dimensions such that a certain objective function (e.g. average within-cluster similarity along the relevant dimensions) is optimized.

While the clusters in real datasets can contain an extremely low percentage of relevant dimensions (less than $10 \%$ of all genes in the previous example), it has been reported that most current projected clustering algorithms are unable to identify clusters with such low dimensionality [22]. This is mainly due to their use of objective functions that highly rely on the accuracy of some input parameters, and the use of similarity calculations that involve all dimensions, which may not reflect the real similarity between different objects [22].

In addition, being unsupervised methods, the algorithms make little use of domain knowledge, despite the fact that a small amount of domain knowledge is usually available in some applications. For example, in gene expression datasets, the functions of a small number of genes are usually known to the biologists. In text mining, some docu- 
ment types have well-known keywords that help identify the member documents. In order to better utilize domain knowledge in the clustering process, a number of semisupervised clustering algorithms [9] have been proposed, which we will review in the next section. The basic idea is to use some domain knowledge to guide the clustering process. For example, in a semi-supervised k-means algorithm [18], domain knowledge about the relationships between some objects is used to force the assignment of some object pairs to the same cluster or to different clusters. As reported in many studies (e.g. $[8,17])$, the clustering accuracy can be greatly improved by inputting only a small amount of domain knowledge.

It should be noted that while some domain knowledge is being used, semi-supervised clustering is different from classification (supervised-learning) in that the knowledge being used in semi-supervised clustering may not be suitable or sufficient for classification. For instance, the knowledge needs not be in the form of objects with class labels as required by classification. The amount of knowledge for each class can be so small that is statistically insignificant to build a classifier that captures the general properties of the class, and the input knowledge can be biased towards one side of the class. The input knowledge of semi-supervised clustering also needs not cover all classes, but it is still possible to produce all the corresponding clusters.

Previous studies on semi-supervised clustering have been focused on non-projected clustering, which does not consider the relevance of dimensions. The semi-supervised approach is in fact very useful in projected clustering. For instance, given a small amount of example objects of a cluster (the "labeled objects", e.g. tumor samples known to be of a certain type), the relevant dimensions of the cluster can be estimated by the dimensions along which the objects are significantly close to each other. Similarly, having a dimension specified as relevant to a cluster (a "labeled dimension", e.g. a gene known to be relevant to a tumor type), the cluster members can be estimated from regions with unexpectedly high object densities.

Semi-supervised clustering also has an important application in handling datasets that have multiple possible groupings. For example, in cancer study, patients can be grouped by their response to a certain treatment, or by the risk of having cancer recurrence. Unsupervised methods can only produce a single set of clusters, which may correspond to only one of the groupings, or even none of them. Using the semi-supervised approach, by supplying different input knowledge, a single clustering algorithm can be guided to produce both kinds of clusters in different runs.

All the above observations motivate the current study, which has three major contributions:

- Proposing a robust objective function for projected clustering that naturally involves dimension selection in the optimization process.

- Proposing the use of domain knowledge (labeled objects and labeled dimensions) to improve the accuracy of projected clustering.

- Developing a new algorithm that can (theoretically and empirically) detect clusters of extremely low dimensionality, and whose accuracy can be further improved by incorporating some domain knowledge in the clustering process.

In the next section we will review some related work in projected clustering and semi-supervised clustering. In Section 3 we will formally define the semi-supervised projected clustering problem, as well as the assumptions being made in this study. In Section 4 we will describe our new algorithm. Experimental results will be presented in Section 5, together with some observations and discussions. In Section 6 we will summarize the whole study and discuss some future extensions.

\section{Related Work}

\subsection{Projected Clustering}

Existing projected clustering algorithms can be classified into three categories: partitional, one cluster at a time, and hierarchical. The partitional approach PROCLUS [1] is based on the traditional k-medoids approach [13], with a goal of minimizing the average within-cluster dispersion. The distance between different cluster members is computed in the relevant subspace of the cluster, which is determined by measuring the average distance between the medoid and a set of "neighboring objects" that are close to it when all dimensions are considered. The dimensions with the smallest average distances to the medoid of each cluster are selected as the relevant dimensions of the cluster, which form its relevant subspace.

Another partitional method ORCLUS [2] improves PROCLUS by selecting principal components so that clusters not parallel to the original dimensions can also be detected. It also adds a hierarchical part that can potentially reduce the errors due to inaccurate initial object assignments.

A limitation of these partitional methods is the determination of neighboring objects based on similarity calculations that involve all dimensions. Since different members of a cluster may appear to be dissimilar when all dimensions are considered, the neighboring objects of a medoid need not be come from the same real cluster and the relevant dimensions suggested by them could be wrong. Also, as the approaches use an objective function that tends to give better scores when fewer dimensions are regarded as relevant to a cluster [22,24], they require users to supply the average 
number of relevant dimensions per cluster, which is usually unknown to users. If improper values are used, the clustering accuracy can be seriously affected.

The Monte Carlo methods DOC and FastDOC [15] identify projected clusters one after another. To find a cluster, an object is randomly selected as the seed, and some other objects are randomly sampled to determine the relevant subspace of the cluster. A dimension is regarded as relevant to the cluster if all the objects are within a distance $\omega$ from the seed along the dimension. Each cluster is thus a hypercube of width $2 \omega$. The more objects and relevant dimensions a cluster has, the less likely it is formed by chance, and thus it receives a better score. The relative importance between the number of objects and relevant dimensions is controlled by a user parameter $\beta$. The algorithm repeatedly tries different seeds and neighboring objects and returns the cluster with the highest score. Then the whole process will be repeated for a new cluster.

The algorithms perform well when each cluster is in the form of a hypercube and the parameter values are specified correctly, but in many cases these requirements cannot be met and the clustering results are quite unsatisfactory [22]. The number of seeds and neighboring objects required to try can also be so large that causes the algorithms to run for a long time.

The hierarchical algorithm HARP is proposed in [22]. Its basic assumption is that two objects are likely to belong to the same cluster if they are very similar to each other along many dimensions. Clusters are allowed to merge only if they are similar enough in a number of dimensions, where the minimum similarity and minimum number of similar dimensions are controlled by two thresholds. At the beginning, the thresholds are set to some harsh values such that only merges that are very likely to group objects belonging to the same real cluster are allowed. As the relevant dimensions of each cluster becomes more apparent, the threshold values are loosened to allow more merges. The process repeats until the thresholds reach their baseline values, or a target number of clusters is reached.

The method successfully avoids extensive distance calculations that involve all dimensions and user parameters whose values are hard to determine. However, due to the hierarchical nature, the algorithm is intrinsically slow. Also, if the number of relevant dimensions per cluster is extremely low (e.g. $5 \%$ of the dataset dimensionality), the accuracy of HARP may drop as the basic assumption will become less valid due to the presence of large amount of noise values in the dataset.

In summary, most of the existing projected clustering algorithms make use of objective functions whose effectiveness rely greatly on the accuracy of some parameter values that are hard for users to determine. Some of them involve similarity calculations that consider all dimensions, which can be quite misleading when cluster dimensionality is small. A thorough survey of the above algorithms and others proposed for two related problems, namely subspace clustering [3] and biclustering [7], can be found in [24].

\subsection{Semi-supervised Clustering}

A recent trend in machine learning research is to combine the techniques developed for unsupervised learning and supervised learning to handle datasets with partial external information. One of the foci is semi-supervised clustering, which actively uses the available domain knowledge in guiding the clustering process. These methods can be categorized according to the kinds of knowledge being input, the time that the knowledge is input, and the way the knowledge is used to affect the clustering process.

The simplest type of input is labeled objects [4,9]. In some cases, users do not know the exact class labels of objects, but they have some knowledge on which objects should be/should not be put into the same cluster, which can be specified by must-links and cannot-links $[5,6,11,17$, 18]. Some other studies propose the input of classification rules [16], examples of similar objects [20], or even general comments like which cluster a particular object should not be put into [8].

The knowledge can be supplied at different time. It can be supplied before clustering to guide the clustering process $[4,5,6,9,11,17,18,20]$, or after clustering to evaluate the clusters and guide the next round of clustering [8]. Some algorithms can also actively request users to supply some specific information at the most appropriate time $[5,11]$.

There are various ways to use the input knowledge, such as guiding the formation of seed clusters $[4,5,6]$, forcing or recommending some objects to be put in the same cluster or different clusters $[17,18]$, and modifying the objective function $[5,6,9]$, similarity function $[8,20]$ or distance matrix [11].

\section{Problem Definition}

We now formally define the semi-supervised projected clustering problem. We start with the data model. Given a dataset $D$ with $n$ objects and $d$ dimensions, the objects can be partitioned into $k$ clusters $\left\{C_{i}\right\}_{i=1}^{k}$ and a possibly empty set of outliers. We assume each cluster is a random sample of the corresponding hidden class, each of which is associated with a set of relevant dimensions that form a relevant subspace. Denote $D_{j}$ and $C_{i j}$ as the projections of $D$ and $C_{i}$ on a dimension $v_{j}$ respectively. Suppose $v_{j}$ is relevant to a subset $R_{j}$ of the clusters, then for each cluster $C_{i} \in R_{j}$, $C_{i j}$ is a random sample of a local Gaussian population with a small variance $\sigma_{i j}^{2}$. The set of all other projected values on $v_{j}, D_{j}-\bigcup_{C_{i} \in R_{j}} C_{i j}$, is a random sample of a global 
population with a variance $\sigma_{j}^{2}$ much larger than the local Gaussians.

Intuitively, in the relevant subspace of a cluster, the cluster members are on average close to each other, but remote from other objects not in the cluster. Alternatively, given a cluster with an unknown relevant subspace, a dimension is more likely to be relevant to the cluster if the projection of the cluster on the dimension has a smaller variance.

To distinguish the actual clusters due to the hidden classes and the clusters produced by a clustering algorithm, we will call the former the "real clusters" and the later simply the "clusters". We will also call the dimensions determined by a clustering algorithm as relevant to a cluster the "selected dimensions" of it.

The inputs to our semi-supervised projected clustering algorithm are:

- The dataset $D$

- The target number of clusters $k$

- A (possibly empty) set $I^{o}$ of labeled objects (<obj. ID, class label $>$ pairs), each indicates that the object is a member of the class. The set may or may not cover all classes.

- A (possibly empty) set $I^{v}$ of labeled dimensions ( $<$ dim. ID, class label $>$ pairs), each indicates that the dimension is relevant to the class. Each dimension can be specified as relevant to multiple classes. The set may or may not cover all classes.

The outputs of the algorithm are $k$ clusters and their selected dimensions, and a (possibly empty) set of outliers. The goal is to optimize an objective function whose value (the objective score) reflects the quality of the clusters. In the non-projected clustering algorithm k-means [10], the objective function is defined as the total within-cluster squared error. It can be shown that the partition of objects that minimize the function corresponds to the maximum likelihood hypothesis of the above model when there are no irrelevant dimensions [12]. In [1], the objective function is modified for projected clustering such that only relevant dimensions are involved in the distance calculations, and the part of objective score from each cluster is normalized by the number of selected dimensions. Due to the normalization, the function tends to gives better (i.e., smaller) scores for clusters with fewer selected dimensions [22,24], which forces the algorithm to request users to supply the average cluster dimensionality in order not to select only one dimension per cluster. Also, as the function is based on the summation of variances among different dimensions, a worse dimension (one with larger variance) constitutes more to the objective score. This means if some irrelevant dimensions are accidentally selected, the objective score can be dominated by the constituents from the irrelevant dimensions, and it can remain virtually unchanged if some relevant dimensions are deselected.

We therefore designed a new objective function with three goals: 1) it should facilitate the selection of dimensions based on the particular data properties of different clusters and dimensions, 2) its value should be constituted more by relevant dimensions, and 3 ) it should be relatively robust. The function, denoted as $\phi$, is defined as follows:

$$
\begin{aligned}
\phi & =\frac{1}{n d} \sum_{i=1}^{k} \phi_{i} \\
\phi_{i} & =\sum_{v_{j} \in V_{i}} \phi_{i j} \\
\phi_{i j} & =n_{i}-1-\frac{1}{\hat{s}_{i j}^{2}} \sum_{x \in C_{i}}\left(x_{j}-\tilde{\mu}_{i j}\right)^{2} \\
& =\left(n_{i}-1\right)\left(1-\frac{s_{i j}^{2}+\left(\mu_{i j}-\tilde{\mu}_{i j}\right)^{2}}{\hat{s}_{i j}^{2}}\right),
\end{aligned}
$$

where $V_{i}$ is the set of selected dimensions of cluster $C_{i}, n_{i}$ is the size of (number of objects in) $C_{i}, x_{j}$ is the projection of an object $x$ on dimension $v_{j}, \tilde{\mu}_{i j}, \mu_{i j}$ and $s_{i j}^{2}$ are the sample median, mean and variance of the projection of $C_{i}$ on $v_{j}$ respectively, and $\hat{s}_{i j}^{2}$ is the selection threshold whose meaning will be explained later. The objective function $\phi$ is composed of the score components $\phi_{i}$ of each cluster, which in turn is the sum of the score components $\phi_{i j}$ of each selected dimension.

There are three major differences between $\phi$ and the objective function defined in [1]. First, $\phi_{i}$ is not normalized by the number of selected dimensions of $C_{i}$, but instead by the threshold $\hat{s}_{i j}^{2}$. As to be discussed later in this section, this allows the dimension selection procedure to be based on the data properties of $C_{i}$ along $v_{j}$ (design goal \#1). It also avoids the existence of trivial best score when each cluster selects only one dimension. Second, by setting $\hat{s}_{i j}^{2}$ to a value that is always larger than the sample variance $s_{i j}^{2}$ of each selected dimension, $\phi_{i j}$ is always positive, and a better dimension (one with smaller $s_{i j}^{2}$ ) has a larger constituent to $\phi_{i}$ (design goal \#2). A better clustering leads to a higher $\phi$ score, so the goal is to maximize the objective score. Third, within-cluster dispersion is measured by the distance from the cluster median rather than centroid, which makes the function less affected by outliers. We will discuss the robustness of $\phi$ later.

The definition of the objective function leads to the following lemma:

Lemma 1 Given a set of clusters $\left\{C_{i}\right\}_{i=1}^{k}$, the objective function $\phi$ is maximized when all dimensions with $s_{i j}^{2}+$ $\left(\mu_{i j}-\tilde{\mu}_{i j}\right)^{2}$ smaller than $\hat{s}_{i j}^{2}$ are selected and all other dimensions are not selected. 
Proof: To maximize $\phi$, a dimension $v_{j}$ should be selected if $\phi_{i j}$ is positive, and should not be selected if $\phi_{i j}$ is negative, which correspond to the cases where $s_{i j}^{2}+\left(\mu_{i j}-\tilde{\mu}_{i j}\right)^{2}$ is smaller than and larger than $\hat{s}_{i j}^{2}$ respectively.

The following dimension selection procedure follows directly from the lemma:

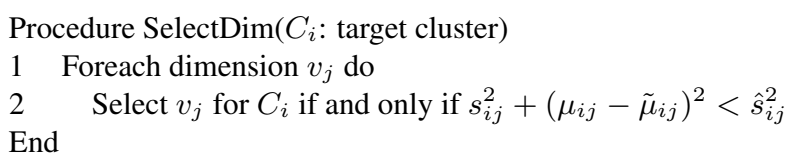

Listing 1: The dimension selection procedure.

$\hat{s}_{i j}^{2}$ is thus called the selection threshold. In the next section we will describe a simple scheme that determines the values of $\hat{s}_{i j}^{2}$, and a more advanced probabilistic-based scheme that can be used when certain conditions are satisfied. In both cases, only one user parameter is required, the value of which is not critical to the clustering accuracy.

In this study we confine the scope by a number of assumptions: 1) clusters are disjoint and axis-parallel, 2) object similarity is based on a distance function, 3) one class corresponds to one cluster and 4) the input knowledge is correct. More information about the assumptions can be found in [23]. The possibility of relaxing some of them will be discussed in Section 6.

\section{The New Algorithm}

The outline of the new algorithm SSPC (SemiSupervised Projected Clustering) is shown in Listing 2. It is a partitional method similar to the k-medoids algorithms [13]. At the beginning it determines some seeds (potential medoids) and each cluster draws a medoid from them. Every object in the dataset is then assigned to the cluster that gives the greatest improvement to the objective score, where the value of $\tilde{\mu}_{i j}$ in Equation 4 is temporarily substituted by the projection of the medoid on $v_{j}$. If an object does not improve the $\phi_{i}$ score of any cluster, it will be put on the outlier list. After assigning all objects, the selected dimensions of each cluster are redetermined and the overall objective score is computed using the actual medians. If the new score is the best one encountered so far, the clusters will be recorded. Otherwise, the best clusters will be restored. A bad cluster is then identified from the current best set of clusters, and a new medoid is selected for it with an attempt to improve the objective score in the next iteration. The medoid of each other cluster is replaced by the cluster median (the virtual object with projected value along each dimension equal to the median of the cluster members) as a medoid could have projected values deviated from the cluster center along some relevant dimensions according to the data model in Section 3. In the next iteration, the values of $\tilde{\mu}_{i j}$ will be substituted by these medians. We will use the term "cluster representative" to call the medoid or median that represents a cluster. After replacing the old cluster representatives, the members of each cluster are removed, and a new iteration of object assignment, score comparison and cluster representatives replacement is carried out. The process repeats until the best objective score remains unchanged for a certain number of iterations.

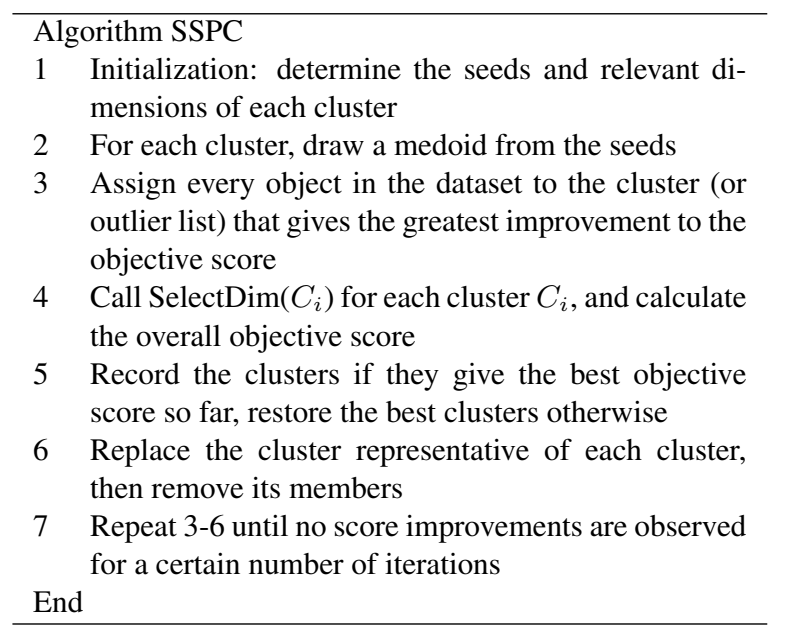

Listing 2: The outline of the SSPC algorithm.

There are three main differences between SSPC and the previous partitional approaches for projected clustering. First, the seeds are determined based on some domain knowledge (labeled objects and labeled dimensions) if supplied, which is potentially more accurate. Second, unlike PROCLUS and ORCLUS where the selected dimensions are determined based on some distance calculations that involve all dimensions, the seeds of each cluster are associated with an estimated set of relevant dimensions determined during initialization. When a seed is picked as the medoid of a cluster, either initially or to replace an old cluster representative, the associated dimensions become the selected dimensions of the cluster. As to be seen later, this process does not rely on distance calculations that involve all dimensions or any user parameters that are critical to the clustering accuracy but whose values are hard to determine. This allows SSPC to identify low-dimensional clusters more accurately. Third, after each iteration, besides replacing a bad cluster representative by a new medoid, other cluster representatives are also replaced by the cluster medians to avoid problems due to the potential biased projected values of the medoids as discussed before.

In the coming subsections some core mechanisms of SSPC will be discussed in detail, followed by an overall analysis of the whole algorithm. 


\subsection{Determining $\hat{s}_{i j}^{2}$}

In order to use the SelectDim procedure and calculate the objective score $\phi$, we need to determine the values of $\hat{s}_{i j}^{2}$. As mentioned in Section 3, $\hat{s}_{i j}^{2}$ should be greater than the sample variance $s_{i j}^{2}$ for all selected dimensions. Since the expected variance of a random sample of a population is the variance of the population, if $s_{i j}^{2}$ is no smaller than the variance of the global population, $\sigma_{j}^{2}$, the members of $C_{i}$ are no more similar to each other along $v_{j}$ than a group of random objects. So, $\sigma_{j}^{2}$ can be viewed as a baseline (maximum) value of $\hat{s}_{i j}^{2}$, whose value can be estimated by the sample variance of $D_{j}$, hereafter denoted as $s_{j}^{2}$.

We propose two schemes to set the actual value of $\hat{s}_{i j}^{2}$. The first scheme is to set it to $m s_{j}^{2}$, where $m \in(0,1]$ is a user parameter. A smaller $m$ tightens the selection criterion. The second scheme is based on a probabilistic reasoning. Users need to specify a value $p$ that bounds the maximum probability that a dimension irrelevant to a cluster is selected by chance. Suppose $C_{i}$ is a cluster to which dimension $v_{j}$ is irrelevant. We can write $p=\operatorname{Pr}\left(s_{i j}^{2}<\hat{s}_{i j}^{2}\right)$. If the sampling distribution of $s_{i j}^{2}$ has a known probability density function (PDF), the value of $\hat{s}_{i j}^{2}$ can be computed accordingly.

For example, suppose the global populations are Gaussian. Then the random variable $\frac{\left(n_{i}-1\right) s_{i j}^{2}}{\sigma_{j}^{2}}$ has a chi-square distribution with $n_{i}-1$ degrees of freedom. With $p$ specified and $\sigma_{j}^{2}$ approximated by $s_{j}^{2}$, the value of $\hat{s}_{i j}^{2}$ can be computed from the inverse of the cumulative chi-square distribution.

The first scheme is more generic as it does not need to assume the properties of the global populations. But in case the sampling distribution of $s_{i j}^{2}$ has a known PDF, the second scheme is more recommended as parameter $p$ has a stronger intuitive meaning than $m$.

Notice that although $\hat{s}_{i j}^{2}$ can take different values for different $C_{i}$ (when parameter $p$ is used) and $v_{j}$ (in both cases), the objective function and the SelectDim procedure involves only one user parameter whose value is not critical to the clustering accuracy, which makes SSPC quite robust. According to the experimental results to be presented in Section 5, the range of values of $m$ and $p$ that give good clustering results is usually much wider than the range of $l$ values (average cluster dimensionality) used by PROCLUS. In general, some reasonable values (e.g. $0.3 \leq m \leq 0.7$, $0.01 \leq p \leq 0.2$ ) can be used when the user has no ideas on the proper value to use. The value can be tuned down if the clusters appear to have too many selected dimensions, or tuned up if too many objects are discarded as outliers.

\subsection{Initialization}

In the initialization step, SSPC determines the seeds and puts them into different seed groups. Each seed group contains a set of seeds that are expected to come from a single real cluster, and a set of dimensions estimated from the seeds to be relevant to the cluster. Each time a seed of a seed group is picked as the medoid of a cluster, the estimated dimensions of the seed group are used as the selected dimensions of the cluster.

SSPC creates two kinds of seed groups: private and public. For a cluster with input knowledge, it is easier to form an accurate seed group (in terms of both the seeds and the estimated relevant dimensions). A private seed group is thus created, which is solely used by the cluster. All other clusters share some large number of public seed groups, so that medoids can be drawn from different seed group combinations. Whenever a cluster needs to draw a new medoid, it is randomly drawn from its private seed group if it has, or otherwise one of the public seed groups not currently used by other clusters.

The order of seed group creation is important. Having created the seed groups of some clusters accurately, it becomes easier to accurately create the remaining seed groups. This is because objects that are close to the seeds of the previous seed groups in the corresponding subspaces are likely members of those clusters, which need not be considered when determining the seeds of the new seed groups. We will show in Section 4.5 that it is easier to create seed groups accurately for clusters with more input knowledge, which suggests that clusters with more input knowledge should have their seed groups created earlier. Based on the ease of creating accurate seed groups, SSPC creates seed groups in the following order: 1) clusters with inputs in both $I^{o}$ and $I^{v}$, 2) clusters with inputs in $I^{o}$ only, 3) clusters with inputs in $I^{v}$ only, 4) clusters with no inputs. Within each category, clusters with larger amount of inputs are initialized earlier.

We now discuss the details of the seed group creation process for the four cases separately. For the first three cases, a private seed group is created, and we will use $C_{i}$ to denote the target cluster, $G_{i}$ the resulting seed group, and $I_{i}^{o}$ and $I_{i}^{v}$ the sets of labeled objects and labeled dimensions for $C_{i}$ respectively. Since $G_{i}$ contains both a set of objects (the seeds) and a set of estimated relevant dimensions, we will sometimes treat it as a cluster for the sake of discussion.

\subsubsection{Clusters with both kinds of inputs}

In this case, the center of $C_{i}$ is likely to be located near the median of $I_{i}^{o}$. Also, if the set of objects in $I_{i}^{o}$ is viewed as a temporary cluster $C_{i^{\prime}}$, the dimensions with larger $\phi_{i^{\prime} j}$ values are more likely to be relevant to $C_{i}$.

This leads to a two-step process of seed group creation. 
We first identify the seeds in $G_{i}$ to be the objects that are close to the median of $I_{i}^{o}$ along dimensions with large $\phi_{i^{\prime} j}$ values. Then we set the relevant dimensions of the seed group to be those selected by $\operatorname{Select\operatorname {Dim}}\left(G_{i}\right)$ plus the ones in $I_{i}^{v}$.

We developed a mechanism for the first step based on a simple idea. If we form a grid (multi-dimensional histogram) of the whole dataset using a fixed number of dimensions, then if the dimensions are all relevant to $C_{i}$, a cell will be found to contain a large number of objects, which correspond to the center of $C_{i}$ in the subspace. If some of the dimensions are irrelevant to $C_{i}$, the peak density (the highest number of objects among the cells) will be much lower. So, multiple grids are built using different sets of dimensions, and the objects in the peak cell with the highest density are chosen as the members of $G_{i}$.

A set of grid-building dimensions could be simultaneously relevant to multiple clusters, in which case the grid will contain multiple peaks. Since the cluster center is expected to be close to the median of $I_{i}^{o}$, it can be located by performing a localized hill-climbing search starting from the cell that contains the median of $I_{i}^{o}$. The search also solves the problem that the median may be biased towards one side of $C_{i}$.

The number of dimensions used to build each grid should not be too large as the number of cells increases exponentially as the number of building dimensions increases, which makes each cell to have too few objects and creates a heavy computational overhead. Normally a threedimensional grid serves the purpose quite well.

Dimensions with greater chance of being relevant to $C_{i}$ should have higher probabilities of being involved in grid building. Therefore we define a candidate set that includes the dimensions selected by $\operatorname{Select\operatorname {Dim}}\left(C_{i^{\prime}}\right)$ as well as those in $I_{i}^{v}$, where each dimension $v_{j}$ in the set has a probability proportional to the $\phi_{i^{\prime} j}$ value of being selected as a building dimension of a grid.

As $\phi_{i^{\prime} j}$ involves the computation of the sample variance $s_{i^{\prime} j}^{2}, I_{i}^{o}$ should contain at least two objects.

\subsubsection{Clusters with labeled objects only}

The seed group creation process is almost the same as in the previous case, except that only dimensions from SelectDim $\left(C_{i^{\prime}}\right)$ are involved in grid building and only those from $\operatorname{SelectDim}\left(G_{i}\right)$ are set as the relevant dimensions of the seed group.

\subsubsection{Clusters with labeled dimensions only}

In this case, the temporary cluster $C_{i^{\prime}}$ cannot be formed. Only dimensions in $I_{i}^{v}$ are involved in grid building, and each of them has the same probability of being involved.
Without $C_{i^{\prime}}$, there is no starting point for the localized hillclimbing search, so the seeds are chosen from the objects in the absolute peak of the whole grid.

\subsubsection{Clusters with no inputs}

In this case, we cannot build the grids directly due to the lack of input knowledge. An alternative mechanism that makes use of the information of other seed groups is developed. It is similar to the max-min mechanism of PROCLUS [1], which identifies an object whose minimum distance to all the seeds already picked by other seed groups is maximum. Distance calculations are performed in the subspace defined by the relevant dimensions of the seed groups, normalized by the number of dimensions. The identified object is remote from all picked seeds, so there is a good chance that it belongs to one of the clusters whose seed group has not been created. It is thus used to replace the median of $I_{i}^{o}$ in the previous cases as the starting point of the hill-climbing search.

An one-dimensional histogram is then constructed for each dimension of the dataset to measure the object density around the identified object along the dimension. A dimension with high object density around the identified object is likely to be relevant to a cluster that centers around the object along the dimension. The dimension is thus given a high probability of being involved in grid building. With these probabilities determined, the seed group creation process for the case with labeled objects only can be performed.

If all clusters have no input knowledge, the object of the first group is randomly drawn from the dataset.

\subsection{Cluster Representatives Replacement}

To improve the objective score, SSPC needs to identify a bad cluster and replace its cluster representative appropriately. Bad clusters most commonly exist when the medoids of two clusters belong to the same real cluster. As a result, the two clusters will be quite similar, and they compete for the $\phi_{i}$ score of the real cluster. On the other hand, one of the other real clusters will not be represented by any cluster, and most of its members will be put on the outlier list.

To detect the occurrence of this situation, one way is to check if there is a cluster with a very low $\phi_{i}$ score, which is likely the loser of the two competing clusters. Another way is to look for clusters that are very similar. In both cases, a bad cluster can be pinpointed and its cluster representative replaced by a new medoid.

For each other cluster, although the medoid may really be a member of the cluster, it may not be close to the cluster center along some relevant dimensions. As a result some real members located at the other side of the cluster may not be attracted to this cluster. The cluster can be improved by 


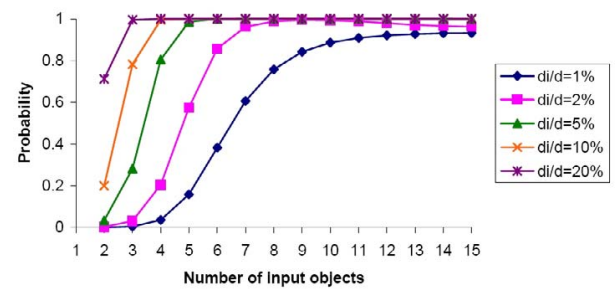

\section{Figure 1. The probability that at least one grid is formed by relevant dimensions only, when only labeled objects are available.}

replacing the medoid with the median of the cluster, which is probably closer to the center of the real cluster.

\subsection{Complexity}

It can be shown [23] that SSPC has a time and space complexity of $O(k n d)$ and $O(n d)$ respectively. The linear complexity makes it more practical to cluster large datasets as compared to some other projected clustering algorithms such as DOC, HARP and ORCLUS.

\subsection{How much input is needed?}

In many real situations the amount of available domain knowledge is very limited. It is therefore important to predict the relationship between the amount of input knowledge and the resulting clustering accuracy, so as to minimize the amount of input knowledge while getting a satisfactory accuracy.

We begin with the case where only labeled objects are available. Suppose a cluster $C_{i}$ receives $\left|I_{i}^{o}\right|$ labeled objects. The objects form a temporary cluster, which is used to determine the grid-building dimensions. We want to estimate the probability that at least one grid is built from dimensions that are really relevant to $C_{i}$ only, which is crucial to the accuracy of the seed group and the clustering accuracy in turn. A closed form formula for the probability is given in [23]. To visualize the change of this value with different input sizes, let us consider some real values to be used in the experiments in Section 5. Suppose $d=3000$, $p=0.01$, each grid involves $c=3$ building dimensions, $g=20$ grids are built for each seed group, and the variance ratio of a local population to the corresponding global population is 0.15 . Figure 1 shows the estimated probabilities that at least one grid is formed by relevant dimensions only with varying $\left|I_{i}^{o}\right|$ and the $\frac{d_{i}}{d}$ ratio, where $d_{i}$ is the number of relevant dimensions of $C_{i}$.

The figure shows that for a fixed $\frac{d_{i}}{d}$ ratio, having more input objects increases the probability of forming a grid

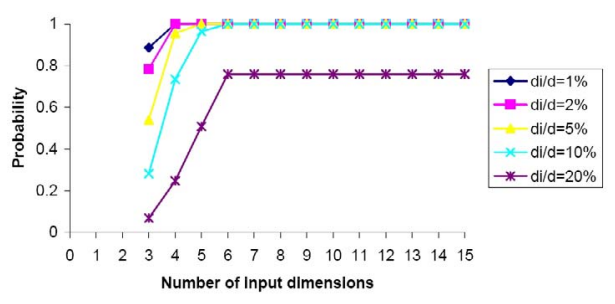

Figure 2. The probability that at least one grid with all $c$ building dimensions being relevant to $C_{i}$ only is formed, when only labeled dimensions are available.

by relevant dimensions only. In addition, each curve has a sharp increase followed by a flattened region. This means users can estimate the smallest amount of input that can lead to a near maximal accuracy. It is an exciting result to see that when $\frac{d_{i}}{d}=5 \%$, only 5 inputs are enough to have an almost $100 \%$ guarantee that a grid will be formed by relevant dimensions only. The figure also shows that for a fixed amount of input, the probability increases as $\frac{d_{i}}{d}$ increases, which suggests that input objects work better when the clusters have more relevant dimensions.

Next, we consider the case with labeled dimensions only. Suppose dimension $v_{j}$ is specified as relevant to a cluster $C_{i}$. If an one-dimensional histogram is built from $v_{j}$, we expect to find a peak at the center of $C_{i}$. If the cell with the highest object density is not close to the center of $C_{i}$, most probably $v_{j}$ is also relevant to another cluster. We want to know the probability that at least one grid has all building dimensions being relevant to $C_{i}$ only. The closed form formula can be found in [23]. Using the same parameter values as before, and setting $k=5$, the estimated probabilities at various $\left|I_{i}^{o}\right|$ and $\frac{d_{i}}{d}$ values are shown in Figure 2.

In general, the more labeled dimensions being supplied, the higher is the chance of forming a grid with all building dimensions being relevant to $C_{i}$ only. The figure also reveals an interesting phenomenon: while labeled objects work better when $\frac{d_{i}}{d}$ is large, labeled dimensions work better when it is small as the chance for a single dimension to be relevant to multiple clusters is small. This suggests that when trying to identify clusters with extremely low dimensionality, which is the main focus of this study, it is more effective to use labeled dimensions as input knowledge.

Both inferences show that a very small amount of input knowledge would enhance the accuracy a lot. Finally, since the two kinds of input complement each other, there is a synergy when they are supplied at the same time, provided the amount of input objects is not so small that causes a large amount of irrelevant dimensions to be used in building the grids. Some empirical results will be presented in the 
next section.

\section{Experiments}

In this section we present various experimental results on SSPC and some comparing algorithms. We compare the accuracy of three projected clustering algorithms HARP [22], PROCLUS [1] and SSPC, using the nonprojected k-medoids clustering algorithm CLARANS [13] as reference.

As far as we know, there exists no benchmark datasets that contain projected clusters as low-dimensional as the ones that are of interest in this study. We therefore generate our own synthetic datasets in ways that are similar to some previous studies [1,24], but with the parameters adjusted to produce datasets with the desired properties. We repeated each experiment 10 times, and report only the result that gives the best algorithm-specific objective score.

The performance metric used to evaluate the quality of a clustering result is the Adjusted Rand Index (ARI) [21] that compares the produced clusters by the known real clusters. It measures how similar are the partition of objects according to the real clusters (U) and the partition in a clustering result (V). Denote $a, b, c$ and $d$ as the number of object pairs that are in the same cluster in both $\mathrm{U}$ and $\mathrm{V}$, in the same cluster in $\mathrm{U}$ but not $\mathrm{V}$, in the same cluster in $\mathrm{V}$ but not $\mathrm{U}$, and in different clusters in both $\mathrm{U}$ and $\mathrm{V}$ respectively, ARI is defined as follows:

$$
A R I(U, V)=\frac{2(a d-b c)}{(a+b)(b+d)+(a+c)(c+d)}
$$

The more similar are the two partitions, the larger will be the ARI value. When $U$ and $V$ are identical, the index value will be one. When $\mathrm{V}$ is only as good as a random partition, the index value will be zero.

If input knowledge is involved in a run of SSPC, the labeled objects are removed from the resulting clusters before computing the ARI values in order to eliminate the direct performance gain due to the input objects.

Due to limited space, some figures are omitted, and they can be found in [23].

\subsection{Raw accuracy}

In the first set of experiments we compared the raw accuracy of the algorithms, i.e., the accuracy without input knowledge. A series of synthetic datasets were generated with $n=1000, d=100$ and $k=5$. The actual average dimensionality of the clusters, $l_{\text {real }}$, varies from 5 to 40 , accounting for $5 \%-40 \%$ of the dataset dimensionality. The datasets were generated according to the data model described in Section 3, with the global distributions being

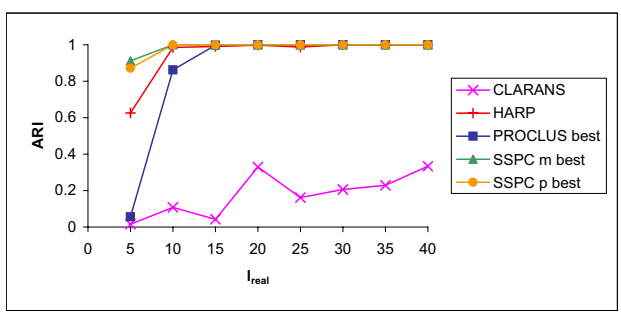

\section{Figure 3. The best raw accuracies of the algo- rithms on datasets with various average clus- ter dimensionality.}

uniform and the local distributions having variances ranging from $1 \%-10 \%$ of the value range of the global distributions.

We set $k$ to 5 for all algorithms, used default parameter values for HARP and CLARANS, and tried different values of the critical parameters of PROCLUS and SSPC. For PROCLUS, we tried 9 different values of $l$ for each dataset. For SSPC, 5 different values of $m$ and $p$ were used for each dataset. The best results (the results with the highest ARI values) after trying different parameter values are shown in Figure 3.

The figure shows that all projected clustering algorithms performed well when the cluster dimensionality is high as compared to CLARANS. When the dataset dimensionality is as low as $5 \%$ of $d$, the performance of all three projected clustering algorithms went down, but SSPC has the mildest performance drop. It is somewhat unexpected that the raw performance of SSPC when parameter $p$ is used is close to the performance when parameter $m$ is used, given the global distributions are actually non-Gaussian. This may due to the fact that except the dimension selection procedure, SSPC makes no assumptions on the global distribution. The performance of the other parts of the algorithm may compensate for the invalid assumptions being made when parameter $p$ is used.

Figure 4 shows the individual clustering results of SSPC and PROCLUS when $l_{\text {real }}=10$, which captures their typical change of accuracy at different parameter values. PROCLUS performed well when the value of $l$ was supplied correctly, but the performance went down as the input moved away from the true value. In contrast, SSPC performed well with the various parameter values being tried, and is thus more robust.

\subsection{Outlier Immunity}

In this set of experiments we studied how SSPC is affected by outliers. A series of synthetic datasets were generated, with the amount of outliers varying from $0 \%$ to $25 \%$. 


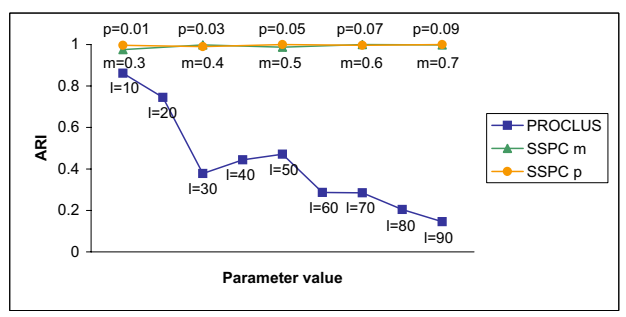

Figure 4. The accuracy of PROCLUS and
SSPC on the dataset with $l_{\text {real }}=10$ using var-
ious parameter values.

The results (figures not shown) show that SSPC has a high noise-immunity, with only moderate accuracy decrease as the amount of outliers increases. The amount of objects detected as outliers also highly resembles the actual amount of outliers in the datasets.

\subsection{Performance with input knowledge}

In this set of experiments, we further lower the average cluster dimensionality and see if the accuracy of SSPC can be improved by input knowledge. We generated a dataset with $n=150, d=3000, k=5$ and $l_{\text {real }}=30$, i.e., $1 \%$ of $d$. The configuration highly resembles a gene expression dataset when the goal is to cluster the samples, and the number of relevant genes of each sample class is as low as $1 \%$ of $d$. We set $m=0.5$, and tried 5 coverage ratios (fraction of clusters receiving inputs), 4 input categories (no inputs, $I^{\circ}$ only, $I^{v}$ only, both), and 8 input sizes. For example, when coverage $=0.6$, both kinds of inputs are supplied and input size $=4,0.6 \times 5=3$ clusters receive input knowledge, each with 4 labeled objects and 4 labeled dimensions. No inputs are supplied for the other 2 clusters.

The inputs are drawn randomly from the real cluster members and relevant dimensions. Each point in the coming figures is the median of 10 repeated runs with 10 independent sets of inputs.

Figure 5 shows the accuracy of SSPC when coverage $=1$. For reference, the ARI values of HARP and PROCLUS (with correct $l$ value supplied) are 0.17 and 0.08 respectively, which are much lower than the raw accuracy of SSPC (at input size 0). In general, SSPC has a larger accuracy improvement when more inputs are supplied. The accuracy becomes stable with 5 objects and 3 dimensions (which is equal to the default value of $c$, the number of building dimensions per grid). All these observations are consistent with the analysis in Section 4.5. The accuracy of SSPC appears to be more stable with labeled dimensions as inputs. In particular, an accuracy lower than the raw accuracy is observed when only 2 labeled objects are supplied

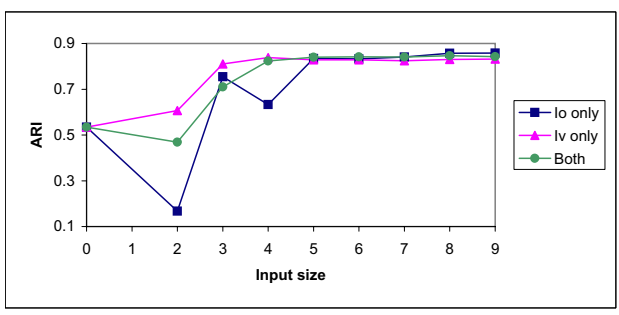

Figure 5. The accuracy of SSPC with various amount of input knowledge when coverage is 1.

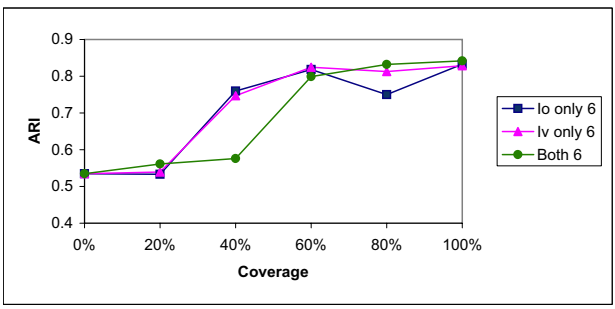

Figure 6. The accuracy of SSPC with various coverage of input knowledge when input size is 6.

to each cluster. This is due to the large probability that the two objects are close to each other along many irrelevant dimensions, which misleads the dimension selection procedure (see Figure 1). In contrast, the probability for a pair of dimensions to be relevant to multiple clusters is much lower due to the low average cluster dimensionality, which results in an observable accuracy improvement when 2 labeled dimensions are supplied.

Figure 6 shows the accuracy of SSPC with changing coverage, when the input size is 6 . There is a general trend of increasing accuracy as the coverage increases. An interesting observation from Figure 6 is that the peak performance is reached at $60 \%$ coverage, which suggests that it is not necessary to input domain knowledge to every cluster. By using the max-min mechanism (Section 4.2.4), clusters with no input knowledge could also locate their cluster centers if the seed groups of the other clusters are created accurately.

\subsection{Data with multiple possible groupings}

As discussed in Section 1, an important application of semi-supervised clustering is to produce different desired clusters based on different input knowledge. In this set of experiments we verify the capability of SSPC in achieving this. We generated two datasets, each with $n=150$, $d=1500, k=5$ and $l_{\text {real }}=30$. The members and 


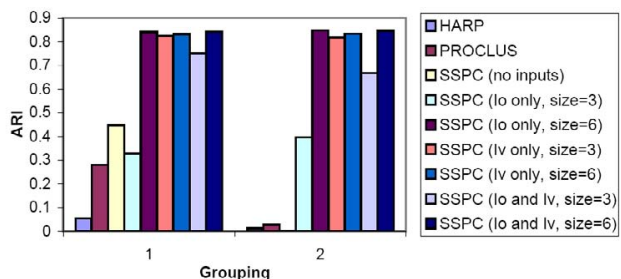

Figure 7. The accuracy of the algorithms on the dataset with two possible groupings.

relevant dimensions of the clusters in the two datasets are independent. We then combined the two datasets to produce a dataset with 3000 dimensions, where the first 1500 come from the first original dataset and the last 1500 come from the second. The average cluster dimensionality thus remains at $1 \%$ of $d$. We then tested the accuracy of HARP, PROCLUS and SSPC on the dataset, with correct $l$ value supplied to PROCLUS. For SSPC, we tested its accuracy in three different scenarios: without inputs (raw accuracy), input based on the knowledge of the first original dataset, and input based on the knowledge of the second original dataset. The ARI values of the algorithms computed from the actual clusters of the two original datasets are shown in Figure 7.

The performance of HARP is seriously affected by the simultaneous existence of two possible groupings. Objects not in the same cluster can be close to each other along many dimensions (as they do belong to the same cluster in the other grouping), which ruin the threshold loosening mechanism of HARP. The performance of PROCLUS is better, but is still not very encouraging. The raw accuracy of SSPC is better than HARP and PROCLUS when evaluated by the first set of clusters, but worse when evaluated by the second set. This shows that without any external inputs, SSPC tends to form clusters that are more similar to the first set. But as some external inputs were supplied, the accuracy of SSPC was significantly improved in both cases. The results confirm the importance of external inputs in guiding the formation of some desired clusters when there are multiple possible groupings.

\subsection{Scalability}

Figures $8 \mathrm{a}$ and $8 \mathrm{~b}$ show the execution time of 10 repeated runs of SSPC with an increasing dataset size $(n)$ and dimensionality $(d)$ respectively, using the execution time of PROCLUS as reference. The figures confirm the linear time complexity of SSPC with respect to both $n$ and $d$. Its speed is comparable to PROCLUS in our implementations.

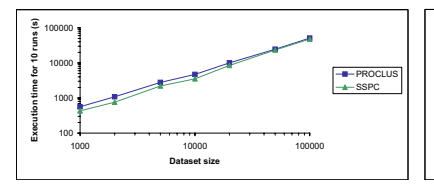

(a) With an increasing $n$.

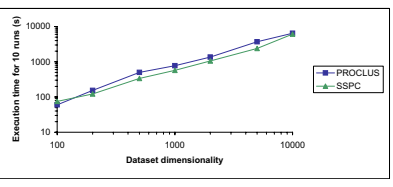

(b) With an increasing $d$.

\section{Figure 8. The execution time of 10 repeated runs of PROCLUS and SSPC.}

\section{Summary and Future Extensions}

In this paper, we have discussed some potential limitations of some existing projected clustering algorithms, including their inability to detect clusters with very low dimensionality, the use of user parameters whose proper values are hard to determine, and the potential accuracy drop when improper parameter values are supplied. We have proposed a new projected clustering algorithm that is robust and is able to detect clusters of extremely low dimensionality as it uses a robust objective function and avoids distance calculations that involve all the dimensions. In addition, we have proposed ways to utilize any available domain knowledge in the form of labeled objects and labeled dimensions. Experimental results show that there is a clear accuracy improvement when some input knowledge is incorporated in the clustering process. The peak performance is readily reached when only a small amount of knowledge is supplied, and when the knowledge covers only some of the classes.

There are some obvious directions for further study. The most important one is to test the new algorithm on some real datasets that are expected to contain projected clusters, such as gene expression profiles. When applying to complex, noisy real data, the data model and objective function may have to be revised according to the observed data properties.

Another direction is to allow incorrect inputs. When inputs could be incorrect, they have to be validated before being used to guide the clustering process, for example by comparing the assumed data model and the observed data values. It is also possible to study fuzzy inputs, each of which contains a confidence level that indicates its chance of belonging to a cluster, and/or a quality level that specifies the chance for the object to be of a certain distance from the cluster center.

It is also interesting to study the case where one class corresponds to multiple clusters. In [11], an interesting algorithm is proposed that modifies the distance matrix such that objects of the same class move towards each other to form a single cluster. The more general approach that al- 
lows the formation of multiple clusters per class is not yet fully studied.

\section{References}

[1] C. C. Aggarwal, C. Procopiuc, J. L. Wolf, P. S. Yu, and J. S. Park. Fast algorithms for projected clustering. In ACM SIG$M O D$ International Conference on Management of Data, 1999.

[2] C. C. Aggarwal and P. S. Yu. Finding generalized projected clusters in high dimensional spaces. In ACM SIGMOD International Conference on Management of Data, 2000.

[3] R. Agrawal, J. Gehrke, D. Gunopulos, and P. Raghavan. Automatic subspace clustering of high dimensional data for data mining applications. In ACM SIGMOD International Conference on Management of Data, 1998.

[4] S. Basu, A. Banerjee, and R. Mooney. Semi-supervised clustering by seeding. In Proceedings of the Nineteenth International Conference on Machine Learning, 2002.

[5] S. Basu, A. Banerjee, and R. J. Mooney. Active semisupervision for pairwise constrained clustering. In Proceedings of the SIAM International Conference on Data Mining, 2004.

[6] S. Basu, M. Bilenko, and R. J. Mooney. Comparing and unifying search-based and similarity-based approaches to semisupervised clustering. In ICML-2003 Workshop on the Continuum from Labeled to Unlabeled Data in Machine Learning and Data Mining, 2003.

[7] Y. Cheng and G. M. Church. Biclustering of expression data. In Proceedings of the 8th International Conference on Intelligent Systems for Molecular Biology, 2000.

[8] D. Cohn, R. Caruana, and A. McCallum. Semi-supervised clustering with user feedback, 2000. Unpublished.

[9] A. Demiriz, K. P. Bennett, and M. J. Embrechts. Semisupervised clustering using genetic algorithms. In Artificial Neural Networks In Engineering, 1999.

[10] J. A. Hartigan and M. A. Wong. A K-means clustering algorithm. Applied Statistics, 28, 1979.

[11] D. Klein, S. D. Kamvar, and C. D. Manning. From instancelevel constraints to space-level constraints: Making the most of prior knowledge in data clustering. In Proceedings of the Nineteenth International Conference on Machine Learning, 2002.

[12] T. M. Mitchell. Machine Learning. McGraw Hill, 1997.

[13] R. T. Ng and J. Han. Efficient and effective clustering methods for spatial data mining. In 20th International Conference on Very Large Data Bases, September 12-15, 1994, Santiago, Chile proceedings, 1994.

[14] S. L. Pomeroy, P. Tamayo, M. Gaasenbeek, L. M. Sturla, M. Angelo, M. E. McLaughlin, J. Y. H. Kim, L. C. Goumnerovak, P. M. Black, C. Lau, J. C. Allen, D. Zagzag, J. M. Olson, T. Curran, C. Wetmore, J. A. Biegel, T. Poggio, S. Mukherjee, R. Rifkin, A. Califano, G. Stolovitzky, D. N. Louis, J. P. Mesirov, E. S. Lander, and T. R. Golub. Prediction of central nervous system embryonal tumour outcome based on gene expression. Nature, 415:436-442, 2002.
[15] C. M. Procopiuc, M. Jones, P. K. Agarwal, and T. M. Murali. A monte carlo algorithm for fast projective clustering. In ACM SIGMOD International Conference on Management of Data, 2002.

[16] L. Talavera and J. Bejar. Integrating declarative knowledge in hierarchical clustering tasks. In International Symposium on Intelligent Data Analysis, 1999.

[17] K. Wagstaff and C. Cardie. Clustering with instance-level constraints. In Proceedings of the Seventeenth International Conference on Machine Learning, 2000.

[18] K. Wagstaff, C. Cardie, S. Rogers, and S. Schroedl. Constrained k-means clustering with background knowledge. In Proceedings of the Eighteenth International Conference on Machine Learning, 2001.

[19] H. Wang, W. Wang, J. Yang, and P. S. Yu. Clustering by pattern similarity in large data sets. In ACM SIGMOD International Conference on Management of Data, 2002.

[20] E. P. Xing, A. Y. Ng, M. I. Jordan, and S. Russell. Distance metric learning, with application to clustering with side-information. In Advances in Neural Information Processing Systems 15, 2003.

[21] K. Yeung and W. Ruzzo. An empirical study on principal component analysis for clustering gene expression data. Bioinformatics, 17(9):763-774, 2001.

[22] K. Y. Yip, D. W. Cheung, and M. K. Ng. HARP: A practical projected clustering algorithm. IEEE Transactions on Knowledge and Data Engineering, 2004. to appear.

[23] K. Y. Yip, D. W. Cheung, and M. K. Ng. On discovery of extremely low-dimensional clusters using semi-supervised projected clustering. Technical Report TR-2004-08, HKU CS, June 2004. http: //www.cs.hku.hk/research/techreps / document/TR-2004-08.pdf.

[24] K. Y. L. Yip. HARP: A practical projected clustering algorithm for mining gene expression data. Master's thesis, The University of Hong Kong, December 2003. http : / / www . CS.hku.hk/ ylyip/papers/thesis.pdf. 